\title{
Velocity-strain relations from hydrostatic measurements on multiple lithologies
}

Vitor Leal de Mello; Guilherme Fernandes Vasquez'; Julio Cesar Ramos Justen'; Marcio José Morschbacher ${ }^{1}$; Filipe Augusto de Souto Borges ${ }^{1}$; Diogo ${ }^{1}$, Folador Rossi', Ricardo Fernandes do Amaral, Jorg Herwanger ${ }^{2}$ and Yves-Marie Leroy ${ }^{3}$

${ }^{1}$ Petrobras

${ }^{2} \mathrm{MP}$ Geomechanics

${ }^{3}$ Total

Copyright 2021, SBGf - Sociedade Brasileira de Geofísica

This paper was prepared for presentation during the $17^{\text {th }}$ International Congress of the Brazilian Geophysical Society held in Rio de Janeiro, Brazil, 16-19 August 2021.

Contents of this paper were reviewed by the Technical Committee of the $17^{\text {th }}$ International Congress of the Brazilian Geophysical Society and do not necessarily represent any position of the SBGf, its officers or members. Electronic reproduction or storage of any part of this paper for commercial purposes without the written consent of the Brazilian Geophysical Society is prohibited.

\begin{abstract}
We investigate the velocity-strain sensitivity on numerous samples from the entire stratigraphic column in the Santos and Campos basins, Offshore Brazil. The samples all measure P-wave velocity as a function of applied effective pressure during hydrostatic loading. We introduce a transform to derive the sensitivity of vertical $\mathrm{P}$-wave velocity to vertical strain, from the observed velocity effective stress relationship. This allows to calculation of $R$-factors, a common parameter to integrated 4D seismic and 4D geomechanical data, from the velocity measurements. The non-linearity in the relationship between P-wave velocity and applied effective stress causes a non-linearity in $R$-factor as a function of initial stress state at which the $R$-factor is evaluated. This naturally explains a depth dependence of $R$-factor in field data. The derived $R$-factor from laboratory data are found to be an order of magnitude larger than those derived from field observations. This is an effect which is commonly observed, and which to date has not been satisfactorily explained. We propose that the relative magnitudes of $R$-factors between different lithologies are a useful diagnostic. We find that shales, marls and pre-salt carbonates have an increased $R$-factor by a factor of approximately four compared to unconsolidated sandstones. Conversely, evaporites (halites and unhydrites) show low $R$-factors of approximately 0.5 of those observed in unconsolidated sandstones.
\end{abstract}

\section{Introduction}

The application of 4D technology in Brazil begun around 22 years ago, although there was a strong commitment of the geophysicists with production seismic monitoring even some years before (Johann et al., 2006). Recently the interest in combining geophysics and geomechanics in 4D seismic studies has increased. This is due to the fact that geomechanical effects can have severe consequences on reservoir permeability, wellbore stability, subsea facilities, and cause environmental issues. As reservoir pore pressure varies during production (due to production or injection), reservoir and surrounding rocks can undergo notable deformation. As a consequence of the deformations the subsurface velocity field changes. Such velocity differences turn the 4D seismic into an important monitoring tool of geomechanical effects, besides the usual important role on saturation monitoring.

Through the so-called $R$ factor it is possible to estimate the vertical strain based on field measurements of $4 D$ timeshifts, or modeling the expected time-shifts based on simulated strain. However, there is a lack of data in the literature regarding $R$ values comprising different rocks in the same basin. We present a formulation that allows us to take advantage of a large dataset of laboratory tests on hydrostatic conditions to support geophysical and reservoir geomechanics integration in the Santos and Campos basins. The dataset comprises of pre-salt carbonates, post-salt carbonates, evaporites and siliciclastic samples. All samples measure P-wave velocity on dry samples under change in effective pressure from atmospheric conditions to effective pressure at reservoir conditions.

The rock physics laboratory from Petrobras can measure elastic-wave velocities under stress at different temperatures and fluid saturation. However, the measurement of dry rock velocities avoids dispersion and attenuation effects. Additionally, these measurements can be done relatively quickly compared to saturated rock velocity measurements. Thus, there is a wide amount of available data available, even though the data is not "ready to use" for dilation factor estimates.

Conversely, the dilation factor could be measured in laboratory, through specifically designed experiments. However, our goal here is to use the large legacy dry rock velocity data. The paper is therefore structured as follows. We first describe the dataset of laboratory measurements. We then derive the theory allowing to estimate $R$-factors from hydrostatic measurements. Lastly, we present the data analysis and place our results into context of reported values of $R-$ factors from the literature.

\section{Dataset}

In the past 30 years numerous laboratory ultrasonic measurements as a function of hydrostatic stress have been conducted on dry rock samples from Brazilian sedimentary basins. To date, the data collection surpasses 8500 plugs and sidewall samples tested. For this work we selected a subsample comprising results for typical lithologies found in the main geological intervals of the Santos and Campos Basins: i) unconsolidated sandstones samples from Oligocene/Miocene; ii) poorly consolidated sandstones from Maastrichtian; iii) consolidated sandstones from Albian/Cenomanian; iv) marls from 
Maastrichtian; v) post-salt marine carbonate from Albian; vi) halite and anhydrite from Aptian, and; vii) pre salt lacustrine carbonate from Aptian. The Tertiary and Late Cretaceus shales and mudstones are underrepresented in the data-base, as they are rarely sampled during coring.

In good quality reservoirs, sample porosities range from approximately $25 \%$ to $35 \%$ for unconsolidated sandstones and $12 \%$ to $24 \%$ for consolidated sandstones. In the carbonates porosities ranges from $20 \%-31 \%$ in post-salt and $7 \%$ to $19 \%$ in pre-salt. These values are lower than $7 \%$ for marls and shales and close to zero for salt samples. Rock compressibility which is directly related to porosity also affects the velocity-strain relation.

\section{Method}

The dilation factor $(R)$ assumes a linear relation between fractional seismic velocity change $\left(\Delta V_{P} / V_{P}\right)$ and vertical strain $\left(\varepsilon_{z}\right)$ (Hatchell and Bourne, 2005; Røste et al., 2005). This model is a simplification of a complex problem involving 4D stress fields and 3D wave propagation phenomena. According to Macbeth et al., (2019) this value remains constant to a given lithology and stress path.

$$
\frac{\Delta V_{P}}{V_{P}}=R \varepsilon_{Z}
$$

One assumption in this formulation is that wave propagation path is near-vertical, and that only vertical strain affects vertical velocity. Both laboratory experiments (e.g. King et al., 1995) and theory (e.g. Sayers, 2005) show that tri-axial stress changes cause anisotropic velocity changes. These theories can also be formulated in terms of strain (Prioul et al., 2004). The angle dependence of velocity changes and the impact of stress/strain path on vertical velocities was discussed in Herwanger and Horne (2009). However, these theories require more complex laboratorial apparatus than measurements under hydrostatic stress changes. Therefore the isotropy assumption and the $R$-factor model are still most popular model (or concept) for connecting 4D seismic and reservoir geomechanics. Thus, we re-state our aim to make use of the available data of measurements under hydrostatic conditions and transform these to estimates of $R$ for different stress/strain paths.

As we are dealing with hydrostatic (or isotropic) bulk compression, for an isotropic rock it is reasonable to assume, also, that the deformation is isotropic, that is:

$$
\varepsilon_{V}=\varepsilon_{x}+\varepsilon_{y}+\varepsilon_{z}=3 \varepsilon_{z}
$$

From bulk modulus definition in a linear elastic media, the vertical strain at hydrostatic condition relative to the initial stress state can be written as:

$$
\varepsilon_{z}=\frac{\Delta l(P)}{l\left(P_{0}\right)}=\frac{-\Delta P}{3 K}
$$

Where, $\Delta P$ is the change on effective pressure, $K$ is the bulk modulus, $l\left(P_{0}\right)$ is the rock sample length at atmospheric pressure $P_{0}$ and $\Delta l(P)$ is the length variation of the rock sample at a given pressure $P$ with respect to $P_{0}$.

Because the fraction change in sample length can be rewritten as:

$$
\frac{\Delta l(P)}{l\left(P_{0}\right)}=d\{\ln [l(P)]\}
$$

We find that:

$$
\int_{P_{0}}^{P} d\{\ln [l(P)]\}=-\frac{1}{3} \int_{P_{0}}^{P} \frac{d P}{K}
$$

Rock compressibilities from laboratory measurements show a non-linear relation as a function of stress. This dependence can be approximated by:

$$
\frac{1}{K(P)}=a P^{b}
$$

Hence, applying equation 6 on equation 5 and solving the integration:

$$
[l(P)]_{P_{0}}^{P}=-\frac{1}{3}\left[\frac{a}{b+1} P^{b+1}\right]_{P_{0}}^{P}
$$

Rearranging the terms, we find:

$$
l(P)=l_{0} \exp \left[\theta\left(P_{0}\right)\right] \exp [\theta(P)]
$$

Where

$$
\theta\left(P_{e f f}\right)=\frac{a}{3(b+1)} P^{b+1}
$$

Thus, calculating $l$ using equation 8 for atmospheric condition, monitor effective pressure $\left(P_{m}\right)$, a reference pressure equivalent to in-situ condition $\left(P_{r e f}\right)$ and combining equations 1 and 3 , we can compute $R$ from:

$$
\frac{V_{P}\left(P_{m}\right)-V_{P}\left(P_{r e f}\right)}{V_{P}\left(P_{r e f}\right)}=R \frac{\exp \left[\theta\left(P_{m}\right)\right]-\exp \left[\theta\left(P_{r e f}\right)\right]}{\exp \left[\theta\left(P_{r e f}\right)\right]}
$$

\section{Results}

Because of the comprehensive dataset it is possible to compare the behavior of different lithologies while considering their respective initial stress states. Ultrasonic compressional velocity measurements as a function of effective stress show that overall velocities range from less than $2000 \mathrm{~m} / \mathrm{s}$ up to $6000 \mathrm{~m} / \mathrm{s}$ (Figure 1). Velocities in sandstones increase with degree of consolidation. The stress sensitivities of elastic wave speeds for all sandstones follow a similar pattern, with a large stress sensitivity at low effective stress and a decreasing stress sensitivity as effective stress increase. Pre-salt and postsalt rocks tend to be less compressible as a function of depth/age which also controls the average porosity. 
Additionally, as compressibility increases the relative velocity change due to stress change also increases (Figure 2a). This effect becomes more evident if one considers the initial stress as the average in-situ stress related to each interval (Figure 2b). In evaporites, compressional velocities exhibit low sensitivity to stress change. Halite samples are more compressible than anhydrite samples, but both are often stiffer than the other sedimentary rocks in the study area.

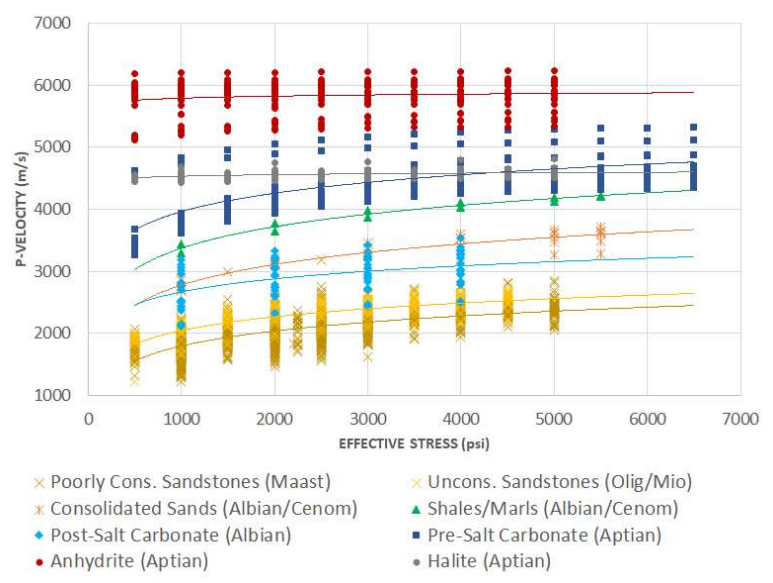

Figure 1: Measurements of ultrasonic compressional velocity as a function of stress for different lithologies: Anhydrite (red), halite (grey), presalt carbonates (dark blue), shales/marls (green), consolidated sandstones (orange) and poorly and unconsolidated sandstones (yellow).

Due to the non-linearity of the velocity-effective stress relations, $R$ values estimated using equation 10 will depend on initial stress state. According to Herwanger (2008) and Bakk et al. (2020) the stress path is one of parameters controlling the $R$-factor. In this work, we consider the initial (or 4D baseline time) stress condition for $R$ computation and assume that any pressure change is an increment (positive or negative) on this reference stress. Figure 2a shows the absolute results of $R$ estimated from hydrostatic tests assuming rocks at an initial stress state representative of the pre-salt interval. When considering lower effective stresses for post-salt rocks, the value of $R$ increases, (Figure 2b). The major differences occur for lithologies with higher $R$ values. The absolute values at the representative initial effective stresses range from approximately 25 to 375 .

It is worth noticing that recent laboratory measurements of $R$, under hydrostatic stress and monitoring rock deformation as well as velocity variation, lead to values between 18 and 44 for saturated sandstone samples (unpublished data).
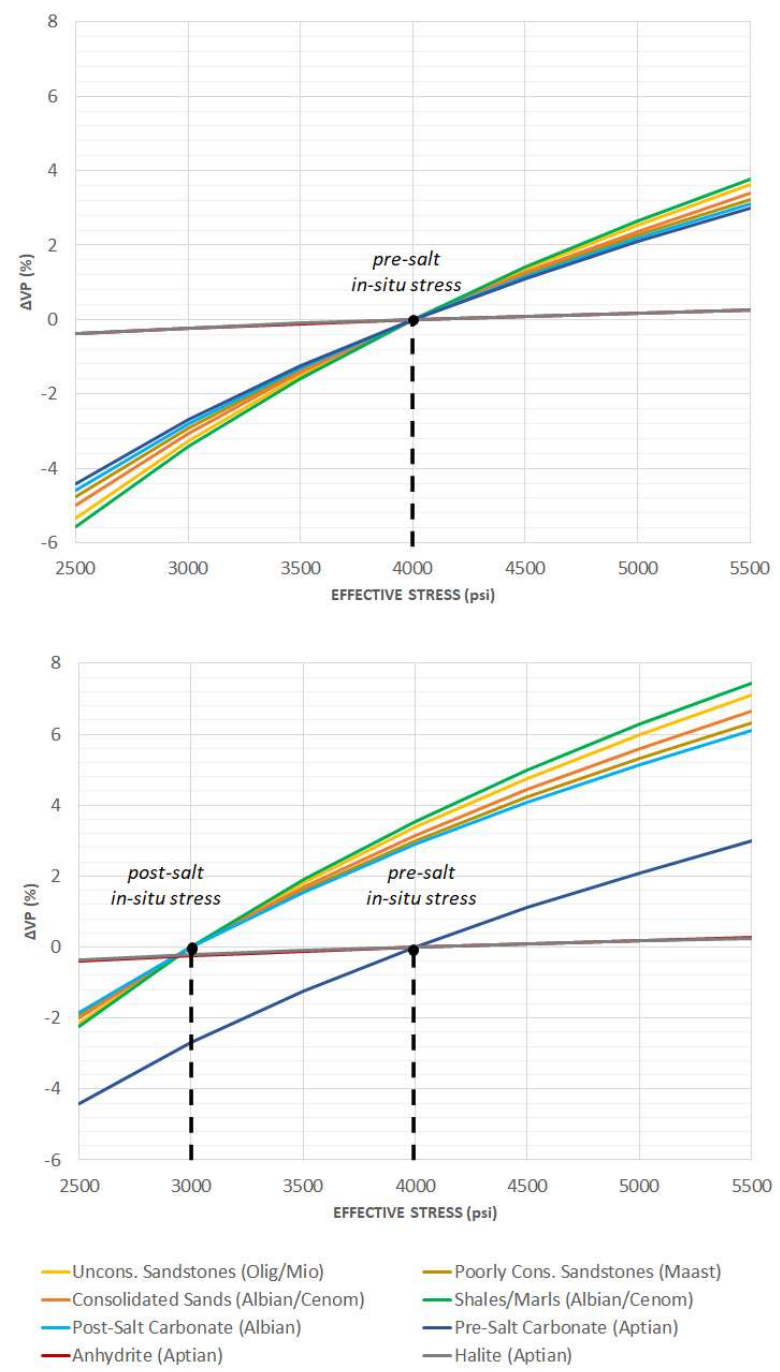

Figure 2: Relative velocity change (\%) as a function of stress for different lithologies assuming: the same initial stress for all lithologies (top) and lower initial stress for post salt rocks (bottom). 

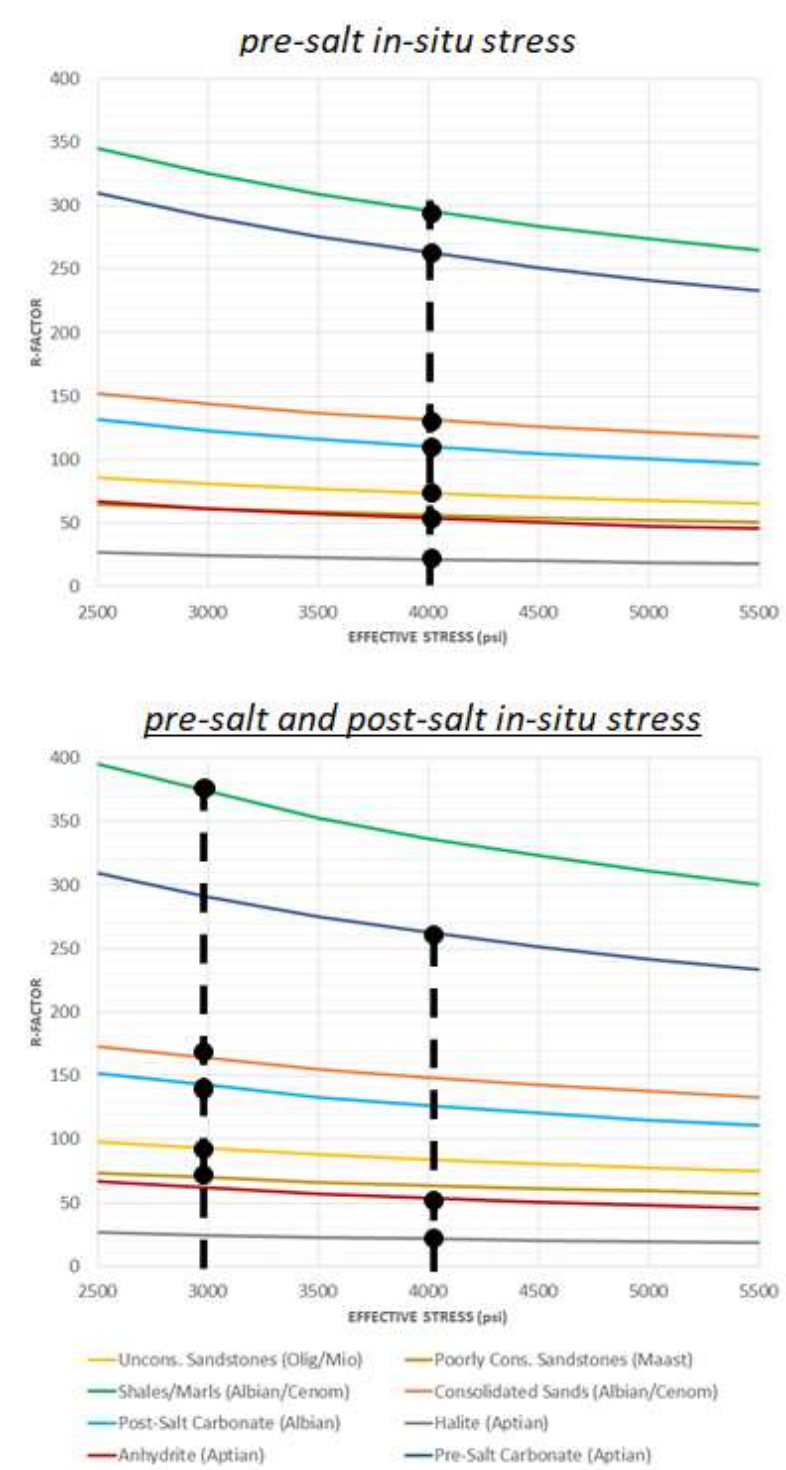

Figure 3: Estimated R-factor for different lithologies as a function of: stress change (top) and combining stress change and initial stress (bottom).

Herwanger and Koutsabeloulis (2011) and MacBeth et al. (2019) summarized published examples of measured $R$ values. It has widely been recognized, that laboratoryderived $R$-factors from ultrasonic measurements are typically higher than those estimated on field scale from seismic monitoring. For example, Herwanger and Koutsabeloulis (2011) re-interpret the shale and sandstone data of Wang (2002) for $R$-factors. They find a range of $\mathrm{R}=7$ to 194 , with a mean value of 83 . This is in line with the observation of $R$-factors in this paper. The difference of laboratory- and field-observation derived $R$-factors makes direct application of laboratory values to field data for strain estimation from observed time-lapse time-shifts difficult, as the predicted strains would be too large. Even though the absolute values may be of little practical relevance, we postulate that the relative magnitude of
$R$-factors are of value for the following reasons: (i) consistency of magnitude of predicted time-lapse changes per lithology, and (ii) to reduce possible errors related to an erroneous assumption of isotropic stress field. (Figure 4).

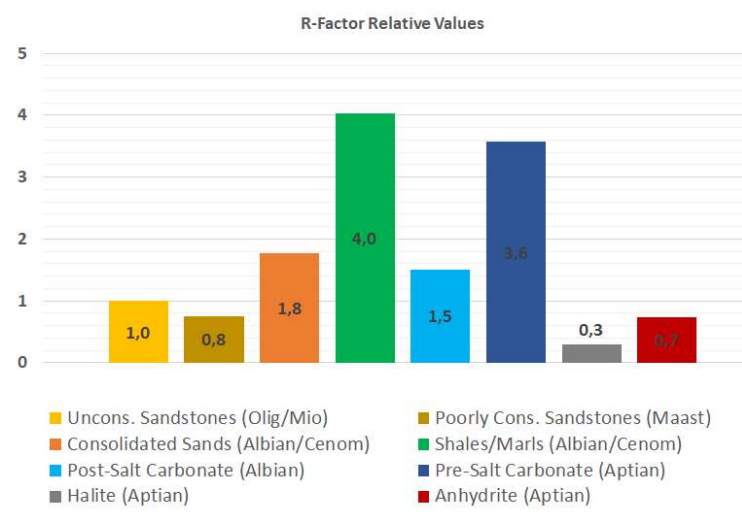

Figure 4: R-factor normalized with respect to unconsolidated sandstone values.

Shales and Marls show the highest $R$ values followed by pre-salt carbonates. One explanation for this behavior in the stiff carbonates might be the fact that the pre-salt carbonates contain microcracks. The presence of microcracks will markedly affect velocity changes due to opening and closing of crack without any relevant deformation. Analogously, shales and marls might similar behavior due to their horizontal bedding, with compliant micro-porosity. In evaporites, both velocity-change and deformation are very small. Their ratio is such that resulting $\mathrm{R}$-factors are also small.

\section{Conclusion}

The characterization of $R$-factor for use in field-wide studies from laboratory measurements is still a challenging task. Firstly, rock frame, mineralogical composition, and the present-day and past 3D stress field contribute to the observed velocity-strain behavior. Secondly, reproducing the in-situ conditions in the laboratory, and measuring simultaneously small deformations and velocities is not trivial. Reproducing in-situ conditions would require investments in new facilities allowing to measure anisotropic velocity changes during tri-axial loading. Lastly, retrieving representative samples is not easy, as neither shales nor faulted and fractured zones are typically cored. It is recommended that coring and testing of such features should be undertaken, as a better characterization of the overburden will not only increase the reliability of 4D seismic interpretation but will also help understanding of the mechanical behavior of the overburden with applications of safe and efficient drilling.

In the meanwhile, we are attempting to make the best use of the large dataset of hydrostatic tests which is presently available. In so doing we are supporting the efforts for better 4D seismic and geomechanics integration. Our two main contributions are (i) to demonstrate how to convert results from hydrostatic measurements to vertical strain 
estimates, and (ii) to introduce the concept of using relative magnitudes of $R$-factor between different lithologies.

The estimated $R$-factor are consistent with other laboratory results found in the literature. A direct application of the values obtained in laboratory in $4 \mathrm{D}$ projects is at present not adequate. The absolute values are an order of magnitude higher than those observed at field scale. Thus, we propose to use lab tests to estimate the proportion between the main geological intervals. In practice, it means that since a representative value for any geological layer is known, either by literature or inference from seismic, the whole subsurface can be characterized in terms of $R$.

The results demonstrate that significant variations in velocity-strain relationships occur when analyzing different lithologies. Porous and unconsolidated rocks are compressible, and they are expected to exhibit large deformation. Therefore, despite significant velocity changes observed in laboratory the $R$-factor is low. In consolidated sandstones and marine carbonates $R$ is approximatelly 1.8 and 1.5 higher than in unconsolidated sandstones, respectively. In pre-salt carbonates, shales and marls from Campos and Santos basins, strain is less affected by stress changes than the ultrasonic velocities. Rock lamination and the presence of cracks can explain the velocity change under stress, but deformation is low due to low compressibility of the material. In the pre-salt carbonates $R$ tend to be 3.6 time greater than unconsolidated sandstones, while shales and marls show an increase of $R$ by a factor of 4.0 compared to sandstones. The salt samples are very stiff, and velocity shows a very low sensitivity to stress change. This scenario result in a decrease of $R$ values by a factor between 0.3 and 0.7 relative values for unconsolidated sandstones.

\section{Acknowledgments}

The authors thank PETROBRAS for authorizing this publication, TOTAL and our colleagues, Josenilda Lonardelli, Paulo Roberto Garcia, Phillip Marchina, Tom Blanchard, Igor Escobar, Elisa Sotelino, Matheus Peres and Leonardo Mesquita for the collaboration on this topic.

\section{References}

Bakk, A., Holt, R. M., Duda, M., \& MacBeth, C. 2020. The Fate of $\mathrm{R}$ in Light of Field Shale Laboratory Tests. In 82nd EAGE Annual Conference \& Exhibition (Vol. 2020, No. 1, pp. 1-5). European Association of Geoscientists \& Engineers. DOI: https://doi.org/10.3997/2214$\underline{4609.202011500}$

Hatchell, P.J., and Bourne, S.J. 2005. Measuring reservoir compaction using time-lapse timeshifts: SEG Technical Program Expanded Abstracts 2005. 2500-2503. DOI: https://doi.org/10.1190/1.2148230

Herwanger, J., 2008, R we there yet?, 70th EAGE Conference and Exhibition, Rome, Italy, June 9-12, paper $\underline{1029}$

Herwanger, J., and Horne, S., 2009, Linking reservoir geomechanics and time-lapse seismics: Predicting anisotropic velocity changes and seismic attributes, Geophysics, 74 (4), W13-W33

Herwanger, J.V. and Koutsabeloulis, N., 2011, Seismic Geomechanics: Building and calibrating geomechanical models using 3D and 4D seismic data, EAGE Publications, ISBN 978-90-73834-10-1

Johann, P.R.S., Thedy, E.A., Gomes, F.A., and Schinelli,M.C., 2006. 4D Seismic in Brazil Experiences in Reservoir Monitoring. 2006 Offshore Technology Conference, Houston, TX, 1-4 May 2006. OTC18400.

King, M.S., Chaudhry, N.A., and Shakeel, A., 1995, Experimental ultrasonic velocities and permeability for sandstones with aligned cracks, Int. J. Rock Mech. Min. Sci. \& Geomech. Abstr., Vol. 32, No. 2, pp. 155-163

MacBeth, C., Mangriotis, M. D., \& Amini, H. 2019. Poststack 4D seismic time-shifts: Interpretation and evaluation. Geophysical Prospecting, 67(1), 331.DOI: https://doi.org/10.1111/1365-2478.12688

Prioul, R., Bakulin, A., and Bakulin, V., 2004, Non-linear rock physics model for estimation of 3-D subsurface stress in anisotropic formations: Theory and laboratory verification, Geophysics, 69(2), 415-425

Sayers, C.M., 2002, Stress-dependent elastic anisotropy of sandstones, Geophysical Prospecting, 50, 85-95

Røste, T., Landrø, M. and Hatchell, P. 2005. Monitoring Overburden Layer Changes and Fault Movements from Time-Lapse Seismic Data: Conference Proceedings, 69th EAGE Conference and Exhibition incorporating SPE EUROPEC 2007, Jun 2007, cp-27-00267 ISBN: 978-9073781-54-2. DOI: https://doi.org/10.3997/2214$\underline{4609.201401685}$ 